\title{
Erratum to: Difficulties in deciding whether to ablate patients with putatively "low-intermediate-risk" differentiated thyroid carcinoma: do guidelines mainly apply in the centres that produce them? Results of a retrospective, two-centre quality assurance study
}

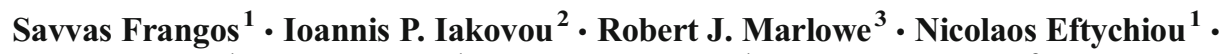

Loukia Patsali ${ }^{1}$ - Anna Vanezi ${ }^{1}$. Androulla Savva ${ }^{1}$ - Vassilis Mpalaris ${ }^{2}$.

Evanthia I. Giannoula ${ }^{2}$

Published online: 2 November 2015

(C) Springer-Verlag Berlin Heidelberg 2015

Erratum to: European Journal of Nuclear Medicine and Molecular Imaging

DOI 10.1007/s00259-015-3124-4

The original version of this article contained a mistake in the reference citation found in Table 5. Instead of ATA 2006, it should have been ATA 2009.

The online version of the original article can be found at http://dx.doi.org/ 10.1007/s00259-015-3124-4.

\section{Savvas Frangos}

savvas.frangos@gmail.com

1 Department of Nuclear Medicine, Bank of Cyprus Oncology Centre, 32 Acropoleos Avenue, Strovolos, Nicosia 2006, Cyprus

2 Department of Nuclear Medicine, Aristotle University, Papageorgiou Hospital, 56403 Thessaloniki, Greece

3 Spencer-Fontayne Corporation, 33 Bentley Avenue, Jersey City, NJ 07304-1901, USA 
Table 5 Classification of the study population in relation to the indications for thyroid remnant ablation according to selected DTC clinical guidelines/ consensus statements

\begin{tabular}{llll}
\hline Category/criterion & EANM 2008 [13] & NCCN Version 2 2014 [15] & ATA 2009 [11] \\
\hline Definite ablation candidates ("indication") & $70.5 \%(237)$ & $19.6 \%(66)$ & $32.7 \%(110)$ \\
Probable ablation candidates ("recommendation") & $10.1 \%(34)$ & $54.8 \%(184)$ & $28.6 \%(96)$ \\
Possible ("selective") ablation candidates ("no indication") & $19.3 \%(65)$ & $25.6 \%(86)$ & $38.7 \%(130)$ \\
\hline
\end{tabular}

The data presented are percentages (number) of patients in relation to the entire cohort ATA American Thyroid Association, EANM European Association of Nuclear Medicine, NCCN National Comprehensive Cancer Network 